\title{
Editorial
}

\section{Novel Technologies and Applications for Construction Materials 2016}

\author{
Gonzalo Martínez-Barrera, ${ }^{1}$ Osman Gencel, ${ }^{2}$ \\ João Marciano Laredo dos Reis, ${ }^{3}$ and Juan José del Coz Díaz ${ }^{4}$ \\ ${ }^{1}$ Laboratorio de Investigación y Desarrollo de Materiales Avanzados (LIDMA), Facultad de Química, Universidad Autónoma del \\ Estado de México, Km. 12 de la Carretera Toluca-Atlacomulco, 50200 San Cayetano, MEX, Mexico \\ ${ }^{2}$ Civil Engineering Department, Faculty of Engineering, Bartin University, 74100 Bartin, Turkey \\ ${ }^{3}$ Theoretical and Applied Mechanics Laboratory (LMTA), Universidade Federal Fluminense, 24220 Rio de Janeiro, RJ, Brazil \\ ${ }^{4}$ Departamento de Construcción, Universidad de Oviedo, 33204 Gijón, Spain
}

Correspondence should be addressed to Gonzalo Martínez-Barrera; gonzomartinez02@yahoo.com.mx

Received 28 December 2016; Accepted 28 December 2016; Published 24 January 2017

Copyright (c) 2017 Gonzalo Martínez-Barrera et al. This is an open access article distributed under the Creative Commons Attribution License, which permits unrestricted use, distribution, and reproduction in any medium, provided the original work is properly cited.

This second special issue shows recent researches in construction technologies as well as in new construction materials. Such investigations involve important aspects, as the use of easy and novel technologies at low costs for improving of physicochemical properties of construction materials, as well as developing environmental friendly and chemically sustainable materials.

The investigations include several topics, for example, the use of by-products in a cement matrix, where thermal conductivity of such composite is measured; for which charcoal from sugarcane bagasse (CSB) was used; the results show that the composite materials reduce thermal conductivities, mainly for higher CSB concentrations ( $15 \%$ by weight). Other research areas involve recycled materials, including glass and zeolite in concrete pavement to mitigate and reduce thermal cracks, as answer to urban heat island (UHI) effect; as we know it is important to reduce pavement high surface temperature in summer to mitigate UHI effect. Moreover, such temperature also affects slab temperature difference, which induces a high possibility of cracking in concrete roads. Also an investigation is concerned about recycled coarse aggregate (RCA) in self-compacting concrete (SCC), which has the potential to reduce both the environmental impact and financial cost associated with this increasingly popular concrete type.

Another important research area covered in this issue is concerned about cementitious composites, with an investigation of the effects of carbon nanotubes in such composites, covering dispersion, implementation, and its effects on the mechanical properties. Concrete structures are often subjected to aggressive aqueous environments which consist of several chemical agents that can react with concrete to produce adverse effects; an investigation is focused in assessing durability of such composites through measurement of surface damage when they are exposed to wastewater; the purpose is to reduce the environmental pollution created by wastewater; which consists of several chemicals such as sulfates, chlorides, and ammonium. Another study is concerned about the effects of capsules on the self-healing efficiency and mechanical properties of cementitious materials; the self-healing due to further hydration of unhydrated cement particles and the efficiency of supply of water by using capsules as a function of capsule dosages and sizes are studied. As we know water is an important factor for developing composite materials; a study is concerned about water-enriched sandy gravel ground as grouting reinforcement applied in river floodplain. Other important materials are mineral aggregates; in this issue investigations for rock texture and composition of it are studied to understand the water vapor diffusion, as well as the vapor sorption in composite materials. Sandstones have been used as construction materials due to their good accessibility and workability. Moreover, they are rocks containing quartz class which can be cemented by secondary 
precipitated quartz or calcite; since most of sandstones are porous, water vapor can penetrate through sandstone constructions.

Some studies about fiber reinforced concrete are described, for example, steel fiber reinforced reactive powder concrete where compressive behavior and mechanical properties are useful for specific application through to stress-strain relationship studies, or steel fiber reinforced lightweight aggregate concrete (SFRLAC) which have many advantages applied in structural engineering, where autogenous and drying shrinkage are measured, considering the effects of types of coarse and fine aggregates with the changes of water-to-binder ratio and volume fraction of steel fiber, as well as a study of a high-performance reactive powder concrete (RPC) and its application in highway bridges, which was prepared with river sand and under $80^{\circ} \mathrm{C}$ steam curing condition and tested in terms of strength, uniaxial compressive stress-strain relation, and flexural load deflection relation.

Metals continue to be important materials for construction, for example, studies of iron ore tailings (IOT) as raw material for manufacturing of Portland cement clinker, where the results showed that the raw meal containing IOT had higher reactivity and burnability than the raw meal containing clay, and the use of IOT did not affect the formation of characteristic mineralogical phases of Portland cement clinker, or studies concerned about physicochemical properties of concrete containing iron ore tailings in order to solve the shortage of natural sand and make full use of industrial waste.

Other types of composite materials are studied, for example, cement-based materials containing combination mineral admixtures, in terms of glass powder (GP), limestone powder (LP), and steel slag powder (SP), where both microstructure and mechanical strength are studied. In order to improve the spalling resistance in lightweight aggregate concrete at high temperature, a study shows two types of modified materials by adopting the surface coating modification method. Mass loss and residual axial compressive strength of the modified concrete after exposure to elevated temperatures were tested. The performance of various plant-produced asphalt concrete mixtures was studied by using different dynamic modulus tests. Finally, colored aggregate use, custom colored cement use, or pigment use with white cement are among the options in order to obtain mixtures in different colors for architectural mortar applications; this experimental study covered freeze thaw resistance properties of mortars.

Gonzalo Martínez-Barrera

Osman Gencel

João Marciano Laredo dos Reis Juan José del Coz Díaz 

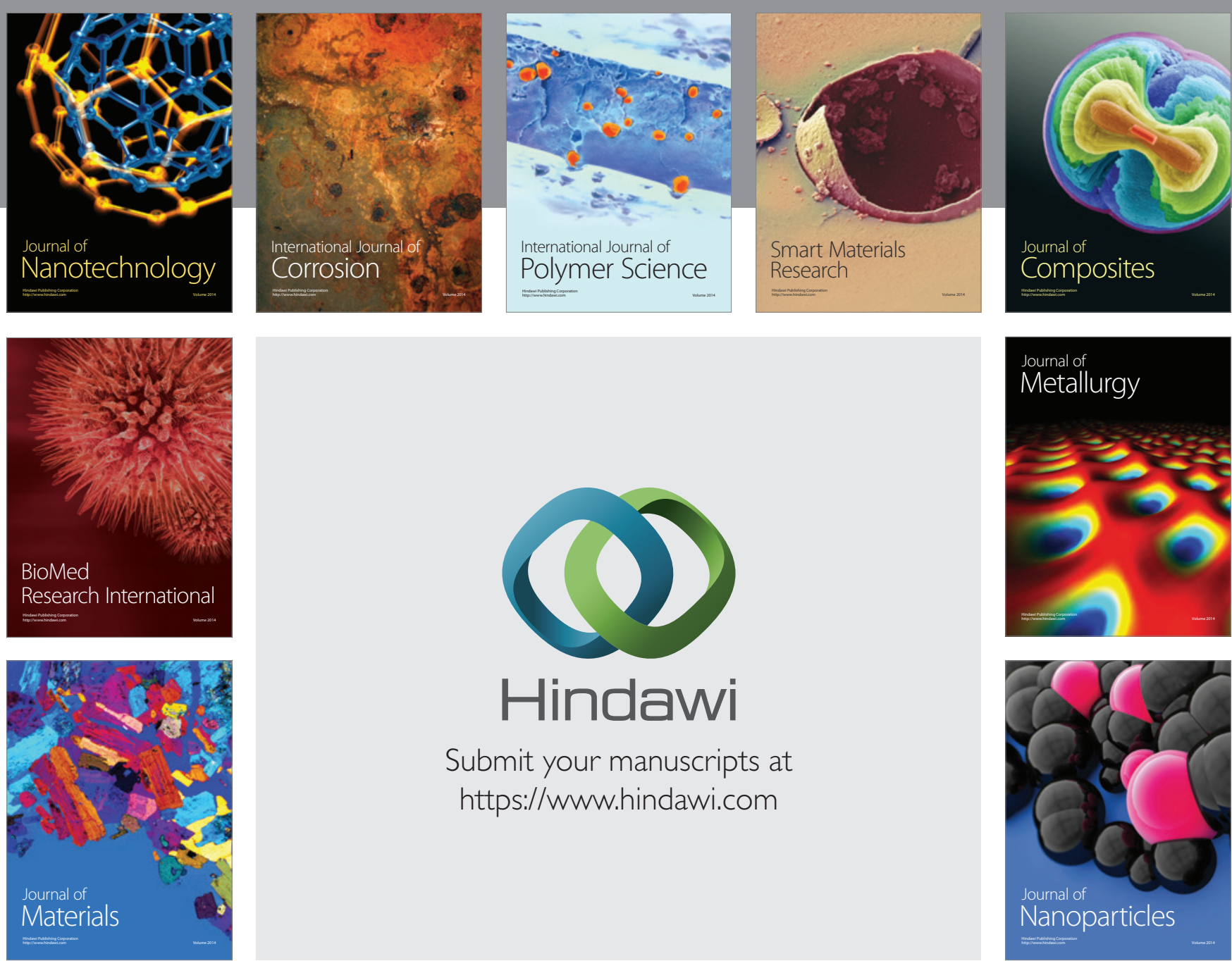

\section{Hindawi}

Submit your manuscripts at

https://www.hindawi.com

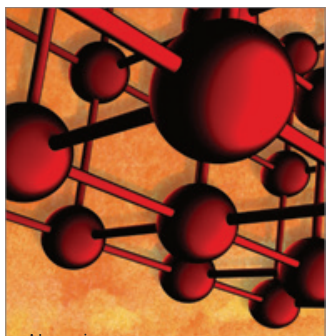

Materials Science and Engineering
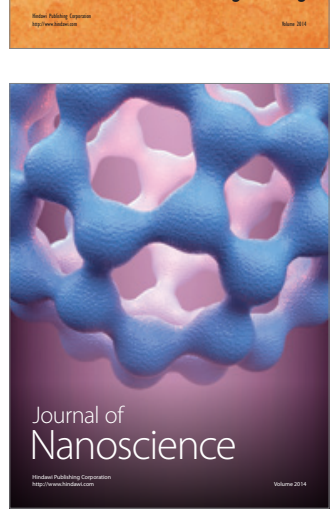
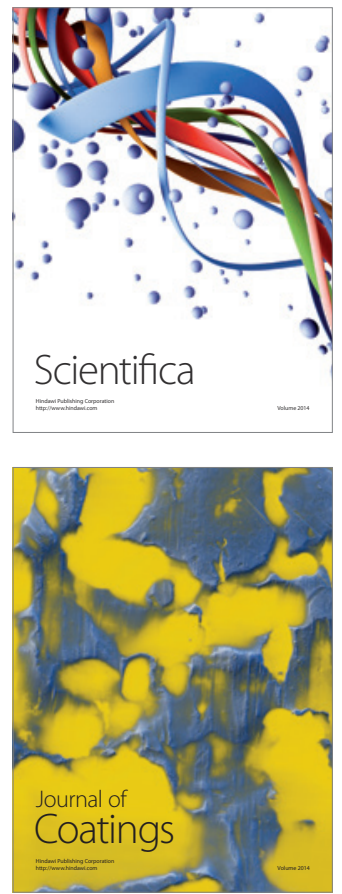
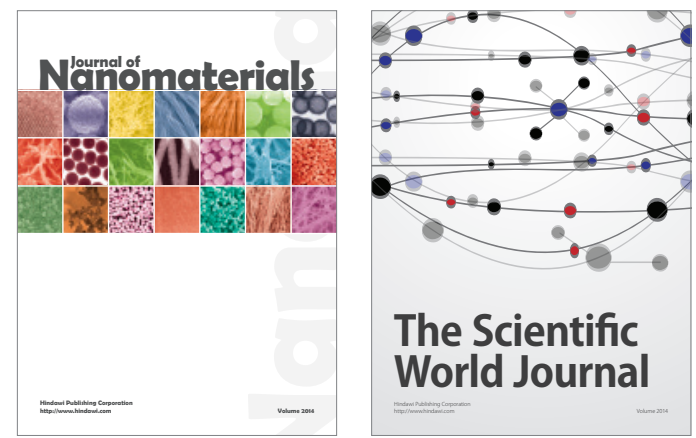

The Scientific World Journal
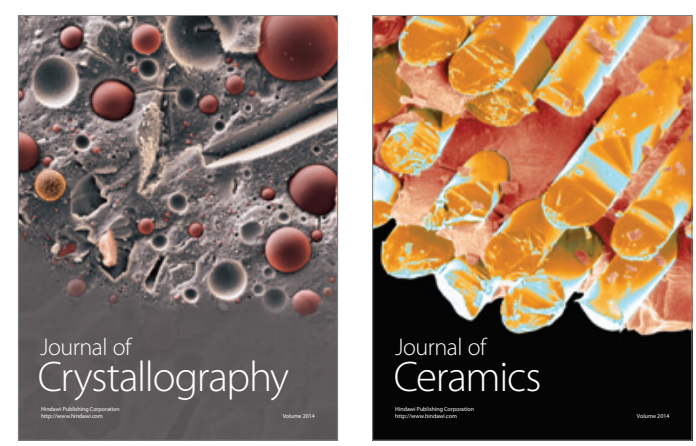
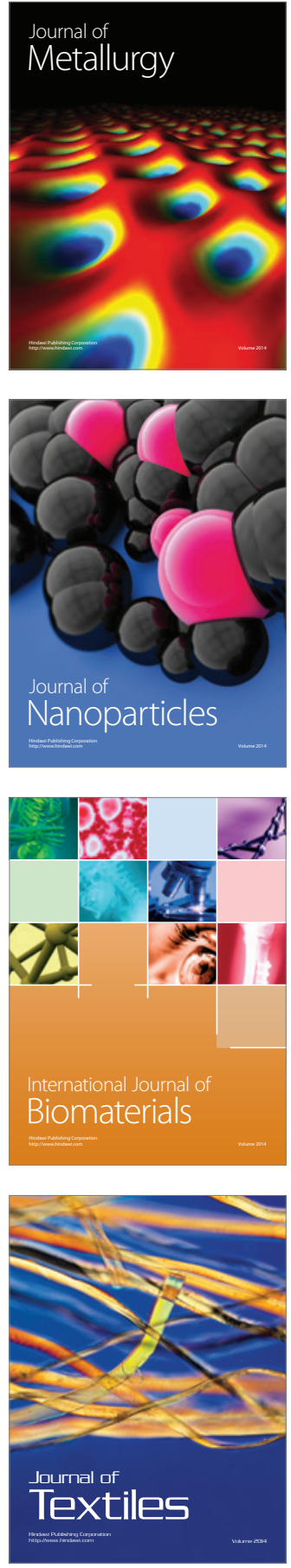